Pacific Journal of Mathematic 


\title{
PARTITIONS OF GROUPS AND COMPLETE MAPPINGS
}

\author{
RICHARD J. FriedLANDER, BASIL GORDON \\ and Peter Tannenbaum
}

\begin{abstract}
Let $G$ be an abelian group of order $n$ and let $k$ be a divisor of $n-1$. We wish to determine whether there exists a complete mapping of $G$ which fixes the identity element and permutes the remaining elements as a product of disjoint $k$-cycles. We conjecture that if $G$ has trivial or noncyclic Sylow 2-subgroup then such a mapping exists for every divisor $k$ of $n-1$. Several special cases of the conjecture are proved in this paper. We also prove that a necessary condition for the existence of such a map holds for every $k$ when $G$ is cyclic.
\end{abstract}

1. Introduction. A complete mapping of a group $G$ is defined to be a bijection $\phi: G \rightarrow G$ such that the mapping $\theta: g \rightarrow g^{-1} \phi(g)$ is also bijective. (Some authors refer to $\theta$, rather than $\phi$, as the complete mapping.) If the permutation $\left(\begin{array}{llll}b_{1} & b_{2} & \cdots & b_{n} \\ c_{1} & c_{2} & \cdots & c_{n}\end{array}\right)$ is a complete mapping of $G$ and $g \in G$, then $\left(\begin{array}{cccc}b_{1} & b_{2} & \cdots & b_{n} \\ c_{1} g & c_{2} g & \cdots & c_{n} g\end{array}\right)$ is clearly also a complete mapping of $G$. By suitable choice of $g$, we can therefore suppose that $b_{n}=c_{n}=1$. Then the complete mapping can be viewed as a permutation $\left(\begin{array}{llll}b_{1} & b_{2} & \cdots & b_{n-1} \\ c_{1} & c_{2} & \cdots & c_{n-1}\end{array}\right)$ of the nonidentity elements of $G$. The permutation $\left(\begin{array}{llll}b_{1} & b_{2} & \cdots & b_{n-1} \\ c_{1} & c_{2} & \cdots & c_{n-1}\end{array}\right)$ is cyclic if and only if it can be written in the form $\left(\begin{array}{lll}a_{1} a_{2} & \cdots & a_{n-1} \\ a_{2} a_{3} & \cdots & a_{1}\end{array}\right)$, where $a_{1}^{-1} a_{2}, a_{2}^{-1} a_{3}, \cdots, a_{n-1}^{-1} a_{1}$ are all distinct. In this case we say that $G$ is an $R$-sequenceable group with $R$-sequencing $a_{1}, a_{2}, \cdots, a_{n-1}$. Thus a group $G$ is $R$-sequenceable if and only if it has a complete mapping which fixes the identity element and permutes the remaining elements cyclically. In [2], we determined several infinite classes of $R$-sequenceable abelian groups (see (1)-(6) below).

In this paper, we generalize the notion of $R$-sequenceability by asking which groups $G$ of order $n$ have the property that, given any regular partition $k+k+\cdots+k$ ( $d$ terms) of $n-1$, there exists a complete mapping of $G$ which fixes the identity element and permutes the remaining elements as a product of $d$ disjoint $k$-cycles. We call such a mapping a k-regular complete mapping of $G$. That is, given any divisor $k$ of $n-1$, a $k$-regular complete mapping of $G$ is a permutation $\left(\begin{array}{llll}b_{1} & b_{2} & \cdots & b_{n-1} \\ c_{1} & c_{2} & \cdots & c_{n-1}\end{array}\right)$ of the nonidentity elements of $G$ whose 
disjoint cycles each have length $k$ and whose quotients $b_{i}^{-1} c_{i}$ also constitute all the nonidentity elements of $G$. If $k=n-1$ then the permutation is cyclic and hence is an $R$-sequencing of $G$.

There are several contexts in which $k$-regular complete mappings arise. For example, 6-regular complete mappings of $Z_{n}$ can be obtained from cyclic Steiner Triple Systems of order $n \equiv 1(\bmod 6)$ [9]. Another occurrence of $k$-regular complete mappings is in connection with a special family of permutation matrices called $I$-matrices [8]. An additional context in which $k$-regular complete mappings arise is in the connection between map coloring and $R$-sequenings of a group [2], [11].

It is well known [6] that if a finite abelian group has a complete mapping, then its Sylow 2-subgroup is either trivial or noncyclic. By a theorem of M. Hall [5], the converse is also true. We conjecture that, given any abelian group $G$ of order $n$ having either trivial or noncyclic Sylow 2-subgroup, there exists a $k$-regular complete mapping of $G$ for each divisor $k$ of $n-1 .^{1}$ We have shown this to be true for $n \leqq 15$, as well as for the following general cases:

(1) $k=n-1, G$ is the cyclic group $Z_{n}$, where $n>1$ is odd.

(2) $k=n-1,(n, 6)=1$ and $n \neq 1$.

(3) $k=n-1, G$ has cyclic Sylow 3 -subgroup, where $n>1$ is odd.

(4) $k=n-1$, the Sylow 2-subgroup of $G$ is $\left(Z_{2}\right)^{m}$, where $m>1$, but $m \neq 3$.

(5) $k=n-1$, the Sylow 2-subgroup $S$ of $G$ is $Z_{2} \times Z_{2^{r}}$ where either

(i) $r$ is odd, or

(ii) $r \geqq 2$ is even and $G / S$ has a direct cyclic factor of order $\equiv 2$ $(\bmod 3)$.

(6) $k=n-1, G=Z_{2} \times Z_{4 r}, r \geqq 1$.

(7) $k$ is any divisor of $n-1, G$ is an elementary abelian $p$ group, $G \neq Z_{2}$.

(8) $k$ is any divisor of $p-1, G$ is an abelian $p$-group $p \neq 2$.

(9) $k=2$ or $(n-1) / 2, G=Z_{n}$, where $n>1$ is odd.

As mentioned above, cases (1)-(6) give $R$-sequencings of $G$ and are proved in [2]. Cases (7) and (8) will be proved in $\S 2$ of this paper and case (9) in $\S 3$.

As a necessary condition for solving the cyclic case for any divisor $k$ of $n-1$, we must be able to divide the nonzero residues $\bmod n$ into $(n-1) / k$ sets, each of cardinality $k$, such that the sum

1 One might also conjecture that there must be a complete mapping corresponding to any partition of $n-1$. However, the cyclic group $Z_{7}$ provides a counterexample, as it has no complete mapping that fixes the identity and permutes the remaining elements as a product of a 4-cycle and a 2-cycle. 
of the elements in each set is $\equiv 0(\bmod n)$. (We use additive notation when the group is cyclic.) We will solve this number theory problem in $\S 4$ of this paper.

2. Abelian $p$-groups. The following theorem gives an infinite family of groups $G$ of order $n$ for which there exists a $k$-regular complete mapping for all divisors $k$ of $n-1$.

THEOREM 1. Suppose $G$ is an elementary abelian p-group of order $n, p$ prime, $G \neq Z_{2}$. Then for any divisor $k$ of $n-1$, there exists a k-regular complete mapping of $G$.

Proof. If $n=p^{m}$, we can write $G=Z_{p} \oplus Z_{p} \oplus \cdots \oplus Z_{p} \quad(m$ times). $G$ is the additive group of $G F\left(p^{m}\right)$, the finite field of $p^{m}$ elements. Let $\alpha$ be a generator of the (cyclic) multiplicative group $G F\left(p^{m}\right)^{*}$ of nonzero elements of $G F\left(p^{m}\right)$. For each divisor $k$ of $n-1$, we define the permutation $\phi$ by $\phi(x)=\alpha^{d} x$, where $d=(n-1) / k$. Since $\alpha^{d}$ has order $k$ in $G F\left(p^{m}\right)^{*}$ and $\alpha^{d}, \alpha^{d}-1 \neq 0$, the permutation $\phi$ is a $k$-regular complete mapping in $G$.

THEOREM 2. Suppose $G$ is an abelian p-group of order $n=p^{m}$, $p$ prime, $p \neq 2$. Then for any divisor $k$ of $p-1$, there exists a $k$-regular complete mapping of $G$.

Proof. If $G=Z_{p^{m}}$ then by a result in [3] there exists a unit $a$ in the ring $Z_{p^{m}}$ such that the mapping $\phi(x)=a \cdot x$ is a $k$-regular complete mapping of $Z_{p^{m}}$.

The result follows by induction and the following observation: If $\phi_{1}$ is a $k$-regular complete mapping of $G_{1}$ and $\phi_{2}$ is a $k$-regular complete mapping of $G_{2}$ then the mapping $(x, y) \rightarrow\left(\phi_{1}(x), \phi_{2}(y)\right)$ is clearly a $k$-regular complete mapping of $G_{1} \times G_{2}$.

3. Cyclic groups. In this section we show the existence of $k$-regular complete mappings of cyclic groups for certain values of $k$.

THEOREM 3. If $k=2$ or $(n-1) / 2$, then there exists a k-regular complete mapping of the cyclic group $Z_{n}$, where $n>1$ is odd.

Proof. The nonzero elements of $Z_{n}$ are $1,2, \cdots, n-1$, the nonzero residues $\bmod n$. For $k=2$, we define the permutation $\phi$ by

$$
\dot{\phi}=(1, n-1)(2, n-2) \cdots\left(\frac{n-1}{2}, \frac{n+1}{2}\right) .
$$

$\phi$ is clearly a product of $(n-1) / 2$ disjoint 2-cycles. The two differences 
occuring in the $j$ th factor of $\rho$ are $j-(n-j)=2 j$ and $(n-j)-j=$ $n-2 j$. As $j$ runs from 1 to $(n-1) / 2$, these differences run through all the nonzero residues $\bmod n$, since $n$ is odd. Thus $\phi$ is a 2-regular complete mapping of $Z_{n}$.

For $k=(n-1) / 2$, we define the permutation $\dot{\phi}$ to be the product of two $k$-cycles $\dot{\phi}_{1}=\left(a_{1}, a_{2}, \cdots, a_{k}\right)$ and $\phi_{2}=\left(-a_{1},-a_{2}, \cdots,-a_{k}\right)$, where the $a_{i}$ are determined as follows:

$$
a_{\imath}=\left\{\begin{array}{l}
(-1)^{i-1}(2 i-1) ; \quad 1 \leqq i \leqq \frac{n+3}{4} \\
(-1)^{i}(2 i-1) ; \quad \frac{n+7}{4} \leqq i \leqq \frac{n-1}{2}
\end{array} \text { if } n \equiv 1 \quad(\bmod 4)\right.
$$

and

$$
a_{i}=\left\{\begin{array}{l}
(-1)^{i-1}(2 i-1) ; 1 \leqq i \leqq \frac{n+1}{4} \\
(-1)^{2}(2 i-1) ; \quad \frac{n+5}{4} \leqq i \leqq \frac{n-1}{2}
\end{array} \quad \text { if } n \equiv 3 \quad(\bmod 4) .\right.
$$

In either case, since $-a_{i}=n-a_{i}$, it is easily checked that the elements $\pm a_{i}, 1 \leqq i \leqq k$, run through all the nonzero residue $\bmod n$. If $n \equiv 1(\bmod 4)$, the differences $a_{i+1}-a_{i}$ in $\phi_{1}$ are $(-1)^{i}(4 i)$ (when $1 \leqq i \leqq(n-1) / 4),(-1)^{i+1}(4 i) \quad($ when $(n+7) / 4 \leqq i \leqq(n-3) / 2), \quad \pm 2$ and 3. A straightforward check shows that, since $n$ is odd, these differences are all distinct and, along with their negatives, run through all the nonzero residues $\bmod n$. The verification for $n \equiv 3(\bmod 4)$ is entirely similar. Thus, in either case, $\phi=\phi_{1} \phi_{2}$ is an $(n-1) / 2$ regular complete mapping of $Z_{n}$.

For the case $k=6$, a 6 -regular complete mapping of $Z_{n}$ for $n \equiv 1$ $(\bmod 6)$ can be constructed from a CIP-neofield $N_{v}$ of order $v \equiv 2$ $(\bmod 6)[1]$ or from an $H P I$-matrix of order $m \equiv 0(\bmod 6)$ [8]. This result can be extended to show the existence of a 6-regular complete mapping of any abelian group of order $\equiv 1(\bmod 6)[14]$.

4. A related number theoretic problem. Let $\phi: G \rightarrow G$ be a complete mapping of $G$, normalized (as in the introduction) so that $\dot{\phi}$ fixes the identity element of $G$. Then as already noted, $\dot{\phi}$ can be regarded as a permutation $\left(\begin{array}{llll}b_{1} & b_{2} & \cdots & b_{n} \\ c_{1} & c_{2} & \cdots & c_{n}\end{array}\right)$ of the elements of $G$ with the property that $a_{i}=b_{i}^{-1} c_{i}(i=1, \cdots, n-1)$ also constitute all the nonidentity elements of $G$. We now decompose this permutation into a product of disjoint cycles, and suppose that $\left(b_{1} b_{2} \cdots b_{r}\right)$ is a typical one of these cycles. Thus $c_{i}=b_{i+1}$ for $i<r$, while $c_{r}=b_{1}$. Hence 
$a_{1} a_{2} \cdots a_{r}=\left(b_{1}^{-1} c_{1}\right)\left(b_{2}^{-1} c_{2}\right) \cdots\left(b_{r}^{-1} c_{r}\right)=\left(b_{1}^{-1} b_{2}\right)\left(b_{2}^{-1} b_{3}\right) \cdots\left(b_{r}^{-1} b_{1}\right)=1 . \quad W e$ have thus proved the following:

THEOREM 4. Suppose that $\phi$ is a complete mapping of $G$ whose associated permutation is the product of disjoint cycles of lengths $r_{1}, r_{2}, \cdots, r_{v}$. Then the elements of $G$ can be partitioned into disjoint subsets $S_{i}$ of cardinality $r_{i}(1 \leqq i \leqq v)$ such that the product of the elements in each subset $S_{i}$ (taken in a suitable order) is 1.

We now specialize to the case where $G=Z_{n}$, the cyclic group of order $n$, and we go over to additive notation. We further suppose that $\dot{\rho}$ is a $k$-regular permutation of $Z_{n}^{*}=Z_{n} \backslash\{0\}$ for some $k>1$, i.e., that $\phi(0)=0$, while the remaining $n-1$ elements of $Z_{n}$ fall into $(n-1) / k$ cycles, each of length $k$. In this case, Theorem 4 asserts that if such a complete mapping $\dot{\phi}$ exists, then the nonzero elements $Z_{n}^{*}$ can be partitioned into $(n-1) / k$ sets of cardinality $k$, where the sum of the elements in each set is $\equiv 0(\bmod n)$. The purpose of this section is to show that this necessary condition for the existence of $\phi$ is always fulfilled as long as $k \mid n-1$ and $n$ is odd. (Of course the condition that $n$ be odd is needed, for only then is the sum of all the elements of $Z_{n}$ congruent to $0(\bmod n)$.) We state this formally as a theorem, although the proof will not be achieved until the end of the section.

THEOREM 5. Suppose $n$ is odd and $k \mid n-1$, where $k>1$. Then the nonzero residues $(\bmod n)$ can be partitioned into $(n-1) / k$ sets of cardinality $k$, so that the sum of the elements of each set is $\equiv 0$ $(\bmod n)$.

We remark that if $k|l|(n-1)$, and that if Theorem 5 has been proved for sets of cardinality $k$, then it also holds for sets of cardinality $l$. Indeed the required sets of cardinality $l$ can be obtained by simply grouping together the sets of cardinality $k$ (in groups of $l / k)$. This reduces the proof of Theorem 5 to the case where $k$ is a prime. For $k=2$ the theorem is trivial, since the required sets are then just $\{1, n-1\},\{2, n-2\}, \cdots,\{(n-1) / 2,(n+1) / 2\}$. For odd values of $k$ we have not been able to take effective advantage of the reduction to primes. Instead we will proceed by mathematical induction through the odd values of $k$. The kernel of the proof is a discussion of the case $k=3$.

When $k=3$, the conditions $k \mid n-1$ and $n$ odd of Theorem 5 are together equivalent to $n \equiv 1(\bmod 6)$. In this case Theorem 5 reduces to 
THEOREM 6. If $n \equiv 1(\bmod 6)$, then the nonzero residues $(\bmod n)$ can be partitioned into $(n-1) / 3$ triples such that the elements of each triple have sum $\equiv 0(\bmod n)$.

This theorem was proved by Skolem [12], [13] for $n \equiv 1$ or 7 $(\bmod 24)$, and by Hanani [7] for $n \equiv 13$ or $19(\bmod 24)$. For the purpose of extending to arbitrary odd $k$, it is necessary to strengthen Hanani's result by proving a conjecture of Skolem [13, p. 274]. We will therefore have to make a fairly elaborate detour. This investigation was originally carried out by one of us (B.G.) in collaboration with W.H. Mills [4]. Related constructions were later carried out by O'Keefe in [10] and Doner in [1]. Since [4] is not in general circulation, we will reproduce the details of the construction here.

Given a set $A=\left\{a_{1}, a_{2}, \cdots, a_{m}\right\}$ of $m$ integers and a set $B=$ $\left\{b_{1}, b_{2}, \cdots, b_{2 m}\right\}$ of $2 m$ integers, we will say that $B$ covers $A$ if $B$ can be partitioned into $m$ disjoint pairs $\left(b_{i_{1}}, b_{j_{1}}\right), \cdots,\left(b_{i_{m}}, b_{j_{m}}\right)$ with $b_{j_{\lambda}}-b_{i_{\lambda}}=a_{\lambda}(1 \leqq \lambda \leqq m)$. We will prove the following conjecture of Skolem:

THEOREM 7. $A_{m}=\{1,2,3, \cdots, m\}$ is covered by $B_{m}=\{1,2,3, \cdots$, $2 m-1,2 m+\varepsilon\}$, where $\varepsilon=0$ if $m \equiv 0$ or $1(\bmod 4)$, and $\varepsilon=1$ if $m \equiv 2$ or $3(\bmod 4)$.

Clearly if $B$ covers $A$, and $\gamma \neq 0$, then any set of the form $\gamma B+\delta=\left\{\gamma b_{1}+\delta, \cdots, \gamma b_{2 m}+\delta\right\}$ covers $\gamma A$ and $-\gamma A$.

Lemma 1. If $u \geqq 1$, the set $F_{u}=\{1,3,5, \cdots, 2 u-1\}$ is covered by $G_{u}=\{1,2,3, \cdots, 2 u\}$.

Proof. An appropriate division of $G_{u}$ into pairs is given by $(i, 2 u+1-i), 1 \leqq i \leqq u$.

Lemma 2. If $u \neq 1$ or 3 , then $F_{u}$ is covered by $H_{u}=\{0,3,4,5, \cdots$, $2 u+1\}$.

Proof. We use induction from $u$ to $u+2$. We have $F_{2}=\{1,3\}$ and $H_{2}=\{0,3,4,5\}$. The pairs $(4,5)$ and $(0,3)$ give a covering of $F_{2}$ by $H_{2}$. For the other initial value of the induction, namely $u=5$, we have $F_{5}=\{1,3,5,7,9\}$ and $H_{5}=\{0,3,4,5,6,7,8,9,10,11\}$. The desired covering of $F_{5}$ by $H_{5}$ is provided by pairs $(7,8),(3,6),(5,10)$, $(4,11)$ and $(0,9)$.

Now assume the lemma true for $u$, and consider $F_{u+2}=$ $\{1,3,5, \cdots, 2 u+3\}$ and $H_{u+2}=\{0,3,4,5, \cdots, 2 u+5\}$. We form the pairs $(0,2 u+3)$ and $(3,2 u+4)$. These give the differences $2 u+3$ 
and $2 u+1$. The remaining elements of $F_{u+2}$ constitute the set $F_{u}$, while the remaining elements of $H_{u+2}$ form the set $\{4,5,6, \cdots$, $2 u+2,2 u+5\}=-1 \cdot H_{u}+(2 u+5)$. By induction (and the above remarks) this set covers $F_{u}$, completing the induction.

LeMma 3. If $u \neq 1,2,4$, then $F_{u}$ is covered by $J_{u}=\{0,2,3, \cdots$, $2 u-2,2 u-1,2 u+1\}$.

Proof. We form the pair $(2,2 u+1)$, which has difference $2 u-1$. The remaining elements of $F_{u}$ constitute the set $F_{u-1}$, while the remaining elements of $J_{u}$ constitute the set $H_{u-1}$ of Lemma 2 . Therefore, Lemma 3 follows from Lemma 2.

Lemma 4. If $u \neq 2$ or 4 , then $F_{u}$ is covered by $K_{u}=\{0,1,4,5$, $6, \cdots, 2 u+1\}$.

Proof. We again use induction from $u$ to $u+2$. We have $F_{1}=\{1\}$ and $K_{1}=\{0,1\}$ so clearly $K_{1}$ covers $F_{1}$. For the other initial value $u=6$, we have $F_{6}=\{1,3,5,7,9,11\}$ and $K_{6}=\{0,1,4$, $5,6,7,8,9,10,11,12,13\}$. A covering of $F_{6}$ by $K_{6}$ is given by the pairs $(6,7),(9,12),(5,10),(1,8),(4,13)$ and $(0,11)$.

Now assume the lemma proved for some integer $u$, and consider $F_{u+2}=\{1,3,5, \cdots, 2 u+3\}$ and $K_{u+2}=\{0,1,4,5,6, \cdots, 2 u+5\}$. We form the pairs $(0,2 u+3)$ and $(1,2 u+2)$ with differences $2 u+3$ and $2 u+1$ respectively. The remaining elements of $F_{u+2}$ constitute $F_{u}$, while the remaining elements of $K_{u+2}$ form the set $\{4,5,6, \cdots$, $2 u+1,2 u+4,2 u+5\}=-1 \cdot K_{u}+(2 u+5)$. By induction, $-1 \cdot K_{u}+$ $(2 u+5)$ covers $F_{u}$, and the proof is complete.

Lemma 5. If $u>1$, then $F_{u}$ is covered by $L_{u}=\{0,1,2,4,5,6, \cdots$, $2 u-1,2 u+1\}$.

Proof. We form the pair $(2,2 u+1)$, which has a difference of $2 u-1$. The remaining elements of $F_{u}$ form the set $F_{u-1}$, while the remaining elements of $L_{u}$ form the set $K_{u-1}$ of Lemma 4 . For $u \neq 3$ or 5, Lemma 5 now follows from Lemma 4. Finally, consider $u=3$ and 5. We have $L_{3}=\{0,1,2,4,5,7\}$ and $L_{5}=\{0,1,2,4,5,6,7,9\}$. The required coverings of $F_{3}$ and $F_{5}$ are given respectively by $(1,2)$, $(4,7),(0,5)$ and $(7,8),(2,5),(1,6),(4,11),(0,9)$.

LeMma 6. If $u \geqq 4$, then $F_{u}$ is covered by $M_{u}=\{0,4,5,6, \cdots$, $2 u+1,2 u+3\}$.

Proof. We form the three pairs $(0,2 u-1),(4,2 u+1),(8,2 u+3)$ 
with differences $2 u-1,2 u-3,2 u-5$ respectively. The remaining elements of $F_{u}$ form the set $F_{u-3}$. If $u=4$, the remaining elements of $M_{4}$ form the set $\{5,6\}$, which covers $F_{1}$. If $u>4$, the remaining elements of $M_{4}$ form the set $\{5,6,7,9, \cdots, 2 u-2,2 u\}=L_{u-3}+5$. In this case the result follows from Lemma 5, and the proof is complete.

We are now ready to prove Theorem 7 . There are six special cases which do not fit into the general pattern. We deal with them separately in the following lemma.

Lemma 7. If $m=2,3,6,7,10$ or 11 , there is a covering of $A_{m}=\{1,2, \cdots, m\}$ by $B_{m}=\{1,2, \cdots, 2 m-1,2 m+1\}$.

Proof. Write $m=2 h+\delta$, where $\delta=0$ or 1 and $h=1,3$ or 5 . We begin by forming the pairs $(1,2)$ and $(2+i, h+2+2 i), 1 \leqq$ $i \leqq m-h$. These pairs have differences $1, h+1, h+2, \cdots, m$. If $h=1$ these pairs constitute the desired covering. If $h=3$ we are left with the problem of covering $\{2,3\}$ by $\{6+2 \delta, 8+2 \delta, 10+2 \delta$, $13+2 \delta\}$, which can clearly be done. If $h=5$, we must cover $\{2,3$, $4,5\}$ by $\{8+2 \delta, 10+2 \delta, 12+2 \delta, 14+2 \delta, 16+2 \delta, 18+2 \delta, 19+2 \delta, 21+2 \delta\}$. The required covering is $(8+2 \delta, 10+2 \delta),(18+2 \delta, 21+2 \delta),(12+2 \delta$, $16+2 \delta),(14+2 \delta, 19+2 \delta)$.

Proof of Theorem 7. Let $m=2 h+\delta$, where $\delta=0$ or 1 . By Lemma 5 , we can assume that $m \neq 2,3,6,7,10$ or 11 . We now use induction on $m$. Note first that $\varepsilon=0$ if $h$ is even, and $\varepsilon=1$ if $h$ is odd. We begin by forming the pairs $(i, h+2 i)$, where $1 \leqq i \leqq$ $m-h=h+\delta$. Then we are left with the problem of covering $\{1,2, \cdots, h\}$ by the union of the sets $\{h+2 \delta+2 i-1 \mid 1 \leqq i \leqq h\}$, $\{3 h+2 \delta+j \mid 1 \leqq j \leqq h-1\}$ and $\{2 m+\varepsilon\}$. Now the set $\{1,2, \cdots, u\}$ can be covered by $\left\{1,2, \cdots, 2 u-1,2 u+\varepsilon^{\prime}\right\}$, where $u=[h / 2]$ and $\varepsilon^{\prime}=0$ if $u \equiv 0$ or $1(\bmod 4), \varepsilon^{\prime}=1$ if $u \equiv 2$ or $3(\bmod 4)$. If $u=0$ this is trivial, if $u=2,3,6,7,10$ or 11 it follows from Lemma 7 , while for all other $u$ it follows from the induction hypothesis. We now distinguish four cases:

Case 1. $h \equiv 0$ or $2(\bmod 8)$. Here $\varepsilon=\varepsilon^{\prime}=0$. Then as just noted, we can cover $\{2,4,6, \cdots, h\}$ by $\{h+2 \delta+2 i-1 \mid 1 \leqq i \leqq h\}$. By Lemma 1 , we can cover $\{1,3,5, \cdots, h-1\}$ by $\{3 h+2 \delta+j \mid 1 \leqq j \leqq h\}$.

Case 2. $h \equiv 1$ or $3(\bmod 8)$. Here $\varepsilon=1, \varepsilon^{\prime}=0$. By assumption we can cover $\{2,4,6, \cdots, k-1\}$ by $\{h+2 \delta+2 i-1 \mid 1 \leqq i \leqq h-1\}$. By Lemma 3 we can cover $\{1,3,5, \cdots, h\}$ by $\{3 h+2 \delta-1\} \cup\{3 h+$ 
$2 \hat{o}+j \mid 1 \leqq j \leqq h-1\} \cup\{2 m+1\}$ unless $h=1,3$ or 7 . Here $h \neq 7$, while $h=1,3$ correspond to $m=2,3,6,7$.

Case $3 . \quad h \equiv 4$ or $6(\bmod 8)$. Here $\varepsilon=0, \varepsilon^{\prime}=1$. By assumption we can cover $\{2,4,6, \cdots, h\}$ by $\{h+2 \delta+2 i-1 \mid 1 \leqq i \leqq h-1) \cup$ $\{3 h+2 \delta+1\}$. By Lemma 2 we can cover $\{1,3,5, \cdots, h-1\}$ by $\{3 h+2 \delta-1\} \cup\{3 h+2 \delta+j \mid 2 \leqq j \leqq h\}$ unless $h=2$ or 6 . Here $h \neq 2$. For $h=6$ a suitable covering of $\{1,2,3,4,5,6\}$ is given by $(23+2 \delta, 24+2 \hat{\delta}),(9+2 \hat{o}, 11+2 \delta),(19+2 \delta, 22+2 \delta),(17+2 \delta, 21+$ $2 \delta),(15+2 \hat{\delta}, 20+2 \hat{\delta}),(7+2 \delta, 13+2 \delta)$.

Case $4 . \quad h \equiv 5$ or $7(\bmod 8)$. Here $\varepsilon=\varepsilon^{\prime}=1$. By assumption we can cover $\{2,4,6, \cdots, h-1\}$ by $\{h+2 \hat{o}+2 i-1 \mid 1 \leqq i \leqq h-2\} \cup$ $\{3 h+2 \delta-1\}$. By Lemma 6 we can cover $\{1,3,5, \cdots, h\}$ by $\{3 h+2 \hat{o}-3\} \cup$ $\{3 h+2 \delta+j \mid 1 \leqq j \leqq h-1\} \cup\{2 m+1\}$ if $h \geqq 7$. There remains $h=5$, which corresponds to $m=10$ and 11 . This completes the proof of Theorem 7.

Proof of Theorem 6. Let $n=6 m+1$. It is trivial to check that the nonzero residues $(\bmod n)$ are the disjoint union of the four sets $A_{m}, B_{m}+m,-A_{m},-\left(B_{m}+m\right)$. By Theorem $7 B_{m}+m$ covers $A_{m}$. This means that $\left(B_{m}+m\right) \cup A_{m}$ is a union of triples $(a, b, c)$ where $a \in A_{m}, b, c \in B_{m}+m$, and $a=b-c$. The triples $(a,-b, c)$ and $(-a, b,-c)$ then exhaust all the nonzero residues $(\bmod n)$, and each one has sum zero.

We turn now to the case $k \geqq 4$ of Theorem 5 . It is convenient to prove it in the following somewhat sharper form.

THEOREM 8. Suppose $n$ is odd and $k \mid n-1$, where $k \geqq 4$. Then the nonzero integer's in the interval $[-(n-1) / 2,(n-1) / 2]$ can be partitioned into $(n-1) / k$ disjoint sets of cardinality $k$, so that the sum of the elements in each set is 0.

Proof. Again we note that this is trivial when $k$ is even, for then we need merely split the interval $[1,(n-1) / 2]$ into $(n-1) / k$ sets of cardinality $k / 2$, and then adjoin to each of these sets the negatives of its elements. Suppose next that $k=5$. The conditions that $n$ is odd and $k \mid n-1$ then yield $n=10 m+1$. We begin by forming the $2 m$ triples $(a,-b, c)$ and $(-a, b,-c)$ constructed in Theorem 6 . If $m \equiv 0$ or $1(\bmod 4)$, the elements of these triples constitute all the nonzero integers in the interval $[-3 m, 3 m]$, and each triple has sum zero. The remaining nonzero integers in the interval $[-(n-1) / 2,(n-1) / 2]=[-5 m, 5 m]$ are symmetric about 0 , 
and there are $2(5 m-3 m)=4 m$ of them. They can therefore be partitioned into $2 m$ pairs of the form $(-j, j)$. To each of the above $2 m$ triples we adjoin one of these pairs. This has the effect of decomposing the nonzero integers of $[-5 m, 5 m]$ into $2 m$ sets of cardinality 5 , where the sum of the integers in each set is zero.

If $m \equiv 2$ or $3(\bmod 4)$, the elements of the triples $(a,-b, c)$ and $(-a, b,-c)$ of Theorem 5 constitute the integers $1,2, \cdots, 3 m-1$, $3 m+1$ and their negatives. The remaining nonzero integers in the interval $[-5 m, 5 m]$ are therefore $3 m$ and $\{v \mid 3 m+2 \leqq v \leqq 5 m\}$, together with their negatives. Since this set is symmetric about 0 , we can again split it into $2 m$ pairs of the form $(-j, j)$ and adjoin one pair to each triple, giving the desired partition of $[-5 \mathrm{~m}, 5 \mathrm{~m}]$ into sets of cardinality 5 .

Exactly the same construction will clearly now carry us from $k$ to $k+2$ for any $k \geqq 5$. It is no longer necessary to distinguish between the various residues of $m(\bmod 4)$, since for $k \geqq 5$, the elements of our $k$-sets constitute all the nonzero integers in $[-(n-1) / 2,(n-1) / 2]$.

We note that the hypothesis in this section that $\phi$ is a $k$-regular permutation can easily be dispensed with, using a very slight modification of the above technique. The only essential requirement is that 0 must be the only fixed point of $\phi$. For simplicity we confined ourselves to the regular case, which seems to be the most interesting in applications. In a later paper the results of this section will be extended to arbitrary groups of odd order.

Note added in proof. A direct, constructive proof of Skolem's conjecture (Theorem 7), appears also in R. O. Davies, "On Langford's problem (II), Math. Gaz., 43 (1959), pp. 253-255. We are grateful to D. G. Rogers (private communication) for pointing out this result as informing us of the following 3-regular complete mapping of $Z_{25}$ : $\phi=(1,8,5)(2,10,11)(3,6,24)(4,14,16)(7,19,17)(9,15,20)(12,23,18)$ $(13,22,21)$. The above partition was obtained by D. G. Rogers and F. W. Roush by means of a computer search.

\section{REFERENCES}

1. J.R. Doner, CIP neofields and combinatorial designs, Ph.D. dissertation, University of Michigan, 1972.

2. R. J. Friedlander, B. Gordon and M.D. Miller, On a group sequencing problem of Ringel, Proc. 9th S.E. Conference on Combinatorics, Graph Theory and Computing, 307-321.

3. R. J. Friedlander, Robert W. Jamison and Peter Tannenbaum, Regular complete mappings of $Z_{n}$, to appear.

4. B. Gordon and W. H. Mills, A theorem on triples of integers, unpublished manuscript.

5. M. Hall, A combinatorial problem on abelian groups, Proc. Amer. Math. Soc., 3 (1952), 584-587. 
6. M. Hall and L.J. Paige, Complete mappings of finite groups, Pacific J. Math., 5 (1955), 541-549.

7. H. Hanani, A note on Steiner triple systems, Math. Scand., 8 (1960), 154-156.

8. D. Hsu, I-matrices and triple systems, I. HP I-matrices and Steiner triple systems, to appear.

9. E. C. Johnsen and T.F. Storer, Combinatorial structures in loops, IV; Steiner triple systems in neofields, Math. Z., 138 (1974), 1-14.

10. E. S. O'Keefe, Verification of a conjecture of T. Skolem, Math. Scand., 9 (1961), 80-82.

11. G. Ringel, Map Color Theorem, Springer-Verlag, New York, 1974.

12. T. Skolem, On certain distributions of integers in pairs with given differences, Math. Scand., 5 (1957), 57-68.

13. - Some remarks on the triple systems of Steiner, Math. Scand., 6 (1958), 273-280.

14. P. Tannenbaum, Abelian Steiner triple systems, Canad. J. Math., 28 (1976), 1251-1268.

Received September 12, 1979 and in revised form April 8, 1980. The first author was supported in part by University of Missouri-St. Louis Faculty Summer Research Fellowship.

The UnIVERSITY OF Missouri

ST LouIs, MO 63121

The University of CALIFornia

LOS ANGELES, CA 90024

AND

The UnIversity of ARIzona

Tucson, AZ 85721 



\section{PACIFIC JOURNAL OF MATHEMATICS}

\section{EDITORS}

DONALD BABBITT (Managing Editor)

University of California

Los Angeles, CA 90024

HUGo RossI

University of Utah

Salt Lake City, UT 84112

C. C. MOORE and ANDREW OGG

University of California

Berkeley, CA 94720
J. DUGUNDJI

Department of Mathematics

University of Southern California

Los Angeles, CA 90007

R. FinN and J. Milgram

Stanford University

Stanford, CA 94305

\section{ASSOCIATE EDITORS}
R. ARENS
E. F. BECKENBACH
B. H. NeumanN
F. WOLF
K. Yoshida

\section{SUPPORTING INSTITUTIONS}

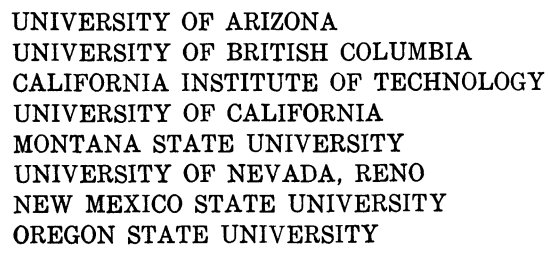

UNIVERSITY OF ARIZONA

UNIVERSITY OF BRITISH COLUMBIA CALIFORNIA INSTITUTE OF TECHNOLOGY

UNIVERSITY OF CALIFORNIA

MONTANA STATE UNIVERSITY

UNIVERSITY OF NEVADA, RENO

NEW MEXICO STATE UNIVERSITY

OREGON STATE UNIVERSITY

\author{
UNIVERSITY OF OREGON \\ UNIVERSITY OF SOUTHERN CALIFORNIA \\ STANFORD UNIVERSITY \\ UNIVERSITY OF HAWAII \\ UNIVERSITY OF TOKYO \\ UNIVERSITY OF UTAH \\ WASHINGTON STATE UNIVERSITY \\ UNIVERSITY OF WASHINGTON
}

The Supporting Institutions listed above contribute to the cost of publication of this Journal, but they are not owners or publishers and have no responsibility for its content or policies.

Mathematical papers intended for publication in the Pacific Journal of Mathematics should be in typed form or offset-reproduced, (not dittoed), double spaced with large margins. Please do not use built up fractions in the text of the manuscript. However, you may use them in the displayed equations. Underline Greek letters in red, German in green, and script in blue. The first paragraph or two must be capable of being used separately as a synopsis of the entire paper. Please propose a heading for the odd numbered pages of less than 35 characters. Manuscripts, in triplicate, may be sent to any one of the editors. Please classify according to the scheme of Math. Reviews, Index to Vol. 39. Supply name and address of author to whom proofs should be sent. All other communications should be addressed to the managing editor, or Elaine Barth, University of California, Los Angeles, California, 90024.

50 reprints to each author are provided free for each article, only if page charges have been substantially paid. Additional copies may be obtained at cost in multiples of 50 .

The Pacific Journal of Mathematics is issued monthly as of January 1966. Regular subscription rate: $\$ 102.00$ a year (6 Vols., 12 issues). Special rate: $\$ 51.00$ a year to individual members of supporting institutions.

Subscriptions, orders for numbers issued in the last three calendar years, and changes of address shoud be sent to Pacific Journal of Mathematics, P.O. Box 969, Carmel Valley, CA 93924, U.S.A Old back numbers obtainable from Kraus Periodicals Co., Route 100, Millwood, NY 10546.

\section{PUBLISHED BY PACIFIC JOURNAL OF MATHEMATICS, A NON-PROFIT CORPORATION}

Printed at Kokusai Bunken Insatsusha (International Academic Printing Co., Ltd.). 8-8, 3-chome, Takadanobaba, Shinjuku-ku, Tokyo 160, Japan. 


\section{Pacific Journal of Mathematics}

Vol. 92, No. $2 \quad$ February, 1981

Bruce Allem Anderson and Philip A. Leonard, Sequencings and Howell designs

Kevin T. Andrews, Representation of compact and weakly compact

operators on the space of Bochner integrable functions . . . . . . . . 257

James Glenn Brookshear, On the structure of hyper-real $z$-ultrafilters . . . . . 269

Frank John Forelli, Jr., A necessary condition on the extreme points of a class of holomorphic functions. II ...................... 277

Richard J. Friedlander, Basil Gordon and Peter Tannenbaum, Partitions of groups and complete mappings ......................... 283

Emden Robert Gansner, Matrix correspondences of plane partitions ......295

David Andrew Gay and William Yslas Vélez, The torsion group of a radical extension ..........................................

André (Piotrowsky) De Korvin and C. E. Roberts, Convergence theorems for some scalar valued integrals when the measure is Nemytskii ...... 329

Takaŝi Kusano and Manabu Naito, Oscillation criteria for general linear ordinary differential equations $\ldots \ldots \ldots \ldots \ldots \ldots \ldots \ldots \ldots \ldots \ldots \ldots \ldots \ldots \ldots \ldots$

Vo Thanh Liem, Homotopy dimension of some orbit spaces .......... 357

Mark Mahowald, $b o$-resolutions . . . . . . . . . . . . . . . . . . . 365

Jan van Mill and Marcel Lodewijk Johanna van de Vel, Subbases, convex

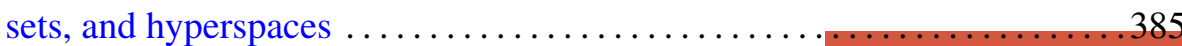

John F. Morrison, Approximations to real algebraic numbers by algebraic numbers of smaller degree $\ldots \ldots \ldots \ldots \ldots \ldots \ldots \ldots \ldots \ldots \ldots \ldots \ldots$

Caroline Series, An application of groupoid cohomology . . . . . . . . . . 415

Peter Frederick Stiller, Monodromy and invariants of elliptic surfaces . . . 433 Akihito Uchiyama, The factorization of $H^{p}$ on the space of homogeneous

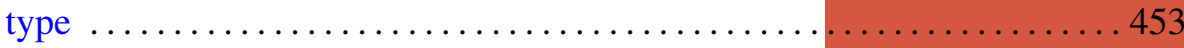

Warren James Wong, Maps on simple algebras preserving zero products.

II. Lie algebras of linear type 